\title{
El cateterismo derecho como herramienta en el diagnóstico de la hipertensión pulmonar
}

\section{Rafael Conde-Camacho ${ }^{\mathrm{a}, \mathrm{b}, *}$ y Jaime Cabrales-Arévalo ${ }^{\mathrm{b}}$}

\author{
a Fundación Neumológica Colombiana, Bogotá, Colombia \\ b Fundación Cardioinfantil-Instituto de Cardiología, Bogotá, Colombia
}

Recibido el 28 de octubre de 2016; aceptado el 5 de julio de 2017

\section{PALABRAS CLAVE \\ Cateterismo derecho; Hipertensión arterial pulmonar; \\ Presión capilar \\ pulmonar; \\ Función ventricular derecha}

\section{KEYWORDS}

Right catheterization;

Pulmonary arterial hypertension;

Pulmonary wedge

pressure;

Right ventricular

function

\begin{abstract}
Resumen La evaluación de la condición hemodinámica del paciente con posible hipertensión pulmonar por medio de cateterismo derecho, ha ganado especial importancia durante los últimos años. Es fundamental contar con información completa al analizar los resultados con el objetivo de aclarar el diagnóstico, establecer el pronóstico, orientar la terapéutica y evaluar la respuesta de la misma. El cateterismo derecho por sí solo no llega al diagnóstico etiológico de la hipertensión pulmonar, pues debe estar enfocado por un estudio previo, organizado y estructurado, en el que se evalúen las causas posibles de la hipertensión enmarcadas en el contexto clínico de cada paciente.

Es fundamental recordar que todas las variables hemodinámicas deben ser evaluadas durante el procedimiento, con el fin de aportar la mayor cantidad de información posible al diagnóstico, tratamiento y seguimiento de este grupo de pacientes con una patología tan catastrófica y con implicaciones de costo tan relevantes para los sistemas de salud, no solo en cuanto al precio de medicamentos sino a la pérdida de años productivos ya que afecta de manera incapacitante a una población joven.

( 2017 Publicado por Elsevier España, S.L.U. en nombre de Sociedad Colombiana de Cardiología y Cirugía Cardiovascular. Este es un artículo Open Access bajo la licencia CC BY-NC-ND (http:// creativecommons.org/licenses/by-nc-nd/4.0/).
\end{abstract}

\section{Catheterisation as a tool in the diagnosis of pulmonary hypertension}

Abstract The assessment of the haemodynamic condition of the patient with possible pulmonary hypertension using right-heart catheterisation, has gained special importance in the last few years. It is essential to have full information on analysing the results, in order to clarify the diagnosis, establish the prognosis, the therapeutic approach, as well as the response to this. Right-heart catheterisation on its own does not provide a diagnosis of the origin of the pulmonary hypertension, since this must be approached by an organised and structured preliminary study, in which the possible causes of the hypertension are evaluated within the clinical context of each patient.

\footnotetext{
* Autor para correspondencia.

Correo electrónico: rconde@neumologica.org (R. Conde-Camacho).
} 
It must be remembered that all the haemodynamic variables should be evaluated during the procedure, with the aim of providing the best quality information possible for the diagnosis, treatment, and follow-up of this group of patients with such a catastrophic disease, and with such important costs for health services, not only as regards the price of the drugs, but also the productive years lost, since it affects and debilitates a young population.

(c) 2017 Published by Elsevier España, S.L.U. on behalf of Sociedad Colombiana de Cardiología y Cirugía Cardiovascular. This is an open access article under the CC BY-NC-ND license (http:// creativecommons.org/licenses/by-nc-nd/4.0/).

El aumento en la presión pulmonar como manifestación patológica está involucrado en un amplio número de enfermedades. Desde el simposio de Eviam en 1998, se estableció la clasificación actual que permitió organizarla en grupos individuales, que comparten hallazgos hemodinámicos similares, hecho que ha servido como guía para orientar y agrupar las alternativas de manejo, de forma tal, que según las características de algunos de estos grupos el fenómeno desencadenante es un componente cardiaco, vascular, respiratorio o sistémico, y por ende, el tratamiento esté orientado al manejo de la condición desencadenante primaria; soló en los grupos 1 y 4 el daño obedece directamente a una alteración intrínseca de la vasculatura pulmonar, constituyéndose en una enfermedad propia de los vasos pulmonares, que genera un impacto directo sobre el corazón derecho y es en esa patología primaria de los vasos pulmonares a donde se dirigen las medidas terapéuticas farmacológicas específicas. Esta diferenciación es vital para lograr un enfoque diagnóstico y terapéutico correcto de la enfermedad arterial pulmonar ${ }^{1}$. Es claro que cada uno de los subgrupos tiene un pronóstico a corto, mediano y largo plazo muy diferente, de ahí que contar con las herramientas y la información adecuada facilite la clasificación del paciente, y permita enfocar las ayudas diagnósticas y establecer de manera más acertada el pronóstico particular de cada caso.

Por otra parte, no existen datos epidemiológicos directos, globales, acerca de la hipertensión pulmonar, por el contrario, múltiples registros que describen las características epidemiológicas de la hipertensión arterial pulmonar. Los registros coinciden con que la hipertensión pulmonar idiopática es una enfermedad rara de uno o dos casos por millón de habitantes en países industrializados, de pronóstico muy pobre sin tratamiento adecuado, con media de supervivencia estimada en 2,8 años, y una tasa de sobrevida de 68, 48 y $34 \%$ al año, y a los 3 y 5 años, respectivamente. La falla cardiaca derecha es la causa más común de muerte ${ }^{2-4}$.

Tras un proceso diagnóstico exhaustivo que parte de una sospecha clínica fundamentada la cual trae como resultado el diagnóstico presuntivo de hipertensión arterial pulmonar se progresa a un ecocardiograma transtorácico, en el que se confirma la sospecha de hipertensión arterial pulmonar con una probabilidad intermedia o elevada; finalmente, es imperativo completar el estudio mediante un cateterismo cardiaco.

El cateterismo cardiaco derecho es el estándar de oro para el estudio del paciente con hipertensión pulmonar, ya que permite la medición directa de presiones en las cavidades cardiacas y en los vasos pulmonares, así como la medición indirecta de los flujos. En la actualidad y con los dispositivos disponibles, es un procedimiento seguro, con una mortalidad relacionada extremadamente baja del $0,055 \%$ y una morbilidad inferior al $1 \%{ }^{6,8}$. El aspecto más crítico de este estudio es que debe realizarse de la forma más apropiada, estructurada y con la mayor rigurosidad posible; adicionalmente, la interpretación de los datos requiere mucha certeza y precisión. En vista de que la presión intratorácica se relaciona estrictamente con la presión atmosférica, la presión intrapleural y el ciclo respiratorio, es vital recordar que todas las variables obtenidas en cavidades derechas o izquierdas, la presión de enclavamiento o cuña pulmonar al igual que las presiones de la arteria pulmonar y aorta deben ser registradas y cuantificadas al final de la expiración ${ }^{5,7,8}$, información particularmente importante en quienes pueden existir variaciones sustanciales entre presiones vasculares inspiratorias y espiratorias, como es el caso de pacientes obesos y con enfermedad pulmonar intersticial ${ }^{5,8-10}$.

De esta manera, el estudio se lleva a cabo con varios objetivos que a su vez permiten dar un orden coherente que conduzca a la interpretación adecuada de los resultados:

1. Confirmar hipertensión pulmonar

2. Cuantificar y categorizarla de forma adecuada

3. Evaluar el compromiso del ventrículo derecho

4. Conocer el pronóstico individual del paciente

5. Definir presencia o no de vasorreactividad pulmonar

Los elementos se resumen en la figura 1.

\section{Confirmar la presencia de hipertensión pulmonar}

Los parámetros hemodinámicos que definen la hipertensión arterial pulmonar (grupo 1 de la clasificación de Niza) son:

- Presión pulmonar arterial media (PAPm) > de 25 mm Hg en reposo

- Presión arterial de enclavamiento pulmonar $(P C P) \leq$ $15 \mathrm{~mm} \mathrm{Hg}$.

- Resistencias vasculares pulmonares $(\mathrm{RVP})>3$ unidades Wood $^{1}$.

Al analizar los componentes de esta definición se hallan elementos vitales en el concepto de hipertensión arterial pulmonar. Ésta en reposo normal es de $14 \mathrm{~mm} \mathrm{Hg}$, con un 


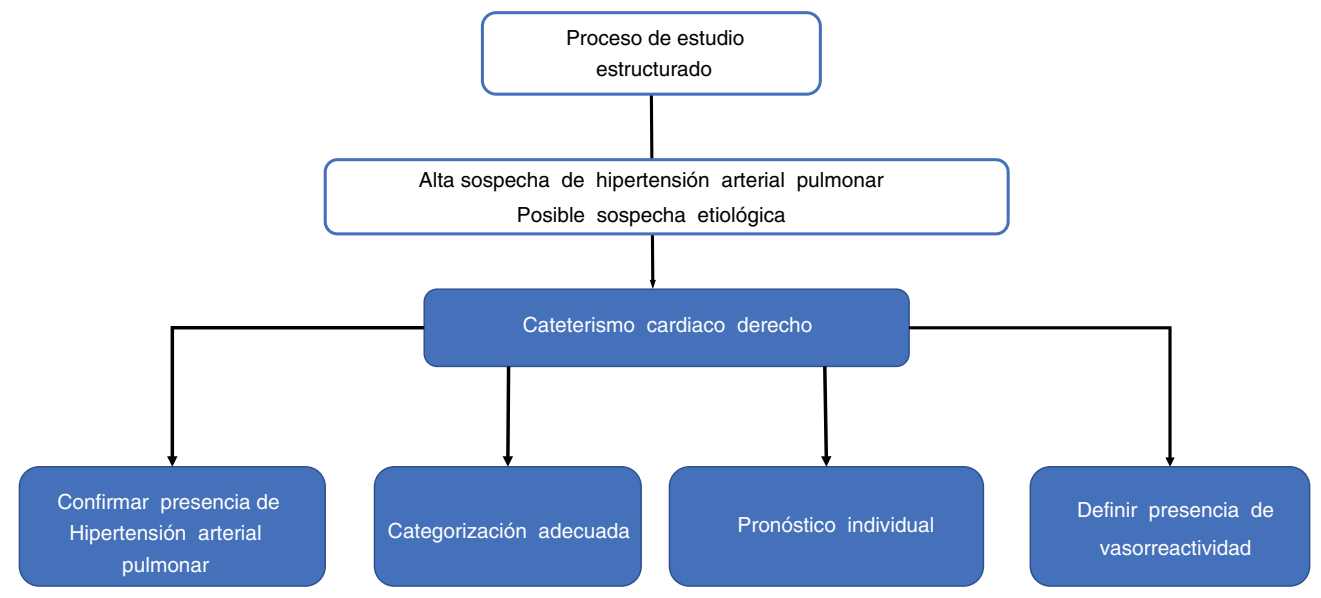

Figura 1 Utilidad del cateterismo cardiaco en la hipertensión pulmonar.

límite superior basado en dos desviaciones estándar de 20,6, que varía un poco con la edad ${ }^{11}$. Un número importante de pacientes tiene valores limítrofes de presiones arteriales medias (definidas como $21-24 \mathrm{~mm} \mathrm{Hg}$ ) en ausencia de enfermedad respiratoria o cardiaca. Aunque en este grupo el incremento de las presiones ha sido identificado como discreto, el pronóstico en el seguimiento es incierto, al igual que el impacto clínico que pueda tener este aumento leve de la presión. En la actualidad no hay evidencia disponible que permita definir si esta población de pacientes requiere un proceso de seguimiento intensivo, o por el contrario puede representar una población relativamente estable en quienes es improbable la progresión de hipertensión arterial pulmonar significativa o establecida ${ }^{12}$. Debido a que ninguna terapia aprobada ha demostrado una reducción significativa en la presión de la arteria pulmonar y por otro lado mejoría en la supervivencia, los principales objetivos en términos de hemodinámica invasiva pretenden evaluar las mejoras en la función cardiaca, en términos de gasto cardiaco, índice cardiaco y presión en la aurícula derecha ${ }^{5}$. Así, el valor neto de la presión media pulmonar permite discriminar la población con enfermedad de la sana, sin que ello tenga una gran implicación en el pronóstico o en la respuesta al tratamiento o que sea fundamental en el proceso de seguimiento ${ }^{13}$.

En cuanto a categorización, tras definir la presencia o no de hipertensión pulmonar, la determinación de la presión de oclusión pulmonar obtenida de la inflación del balón en la punta del catéter crea una oclusión al flujo anterógrado con lo cual se genera un continuo en la columna de sangre distal al catéter que se extiende a las venas pulmonares, permitiendo así estimar la presión en la auricula izquierda, y en ausencia de estenosis o patología significativa de la válvula mitral la presión telediastólica del ventrículo izquierdo. Con la intención de obtener una trasmisión retrógrada de la presión ventricular izquierda a través de la red capilar, la punta del catéter debería ubicarse en un segmento pulmonar donde la presión venosa pulmonar supere a la alveolar, como lo es la zona fisiológica 3. Condiciones tales como hipovolemia, enfermedad parenquimatosa avanzada y presión positiva al final de espiración, pueden hacer que la presión alveolar supere la presión venosa pulmonar creando comportamientos fisiológicos de zonas 1 y 2 , en cuyos casos la presión de oclusión llega a ser un estimativo de la presión alveolar más que la presión de la aurícula izquierda ${ }^{9,14}$.

Las guías actuales recomiendan la utilización de una presión capilar pulmonar (PCP) o presión telediastólica del ventrículo izquierdo (PTDVI) $\leq 15 \mathrm{~mm} \mathrm{Hg}$ para definir la hipertensión pulmonar pre-capilar. Generalmente, en los pacientes con patologías que comprometen las cavidades del lado izquierdo del corazón se obtienen valores más elevados de PCP, de aquí la importancia como elemento diferenciador entre dos grupos totalmente opuestos. Los valores normales de PCP han sido analizados desde los inicios del cateterismo cardiaco y se encuentran en valores de entre 5 y $12 \mathrm{~mm} \mathrm{Hg}$ en voluntarios sanos. Pese a que la PCP normal aumenta ligeramente con la edad, normalmente no se eleva por encima de los $15 \mathrm{~mm} \mathrm{Hg}^{14-18}$.

Los errores en las mediciones de PCP son frecuentes y en muchas ocasiones están relacionados $\operatorname{con}^{14-18}$ :

- No identificación de un trazado amortiguado de la AP.

- No tener en cuenta los efectos de la respiración en las mediciones.

- Cambios en la presión intratorácica.

- Inadecuado posicionamiento del catéter durante la medición (no tomar oximetría que confirme adecuada ubicación ante la imposibilidad de seguimiento fluoroscópico).

- Determinación digital y no manual de la presión de oclusión pulmonar dado que esta tiende a ser inferior que la presión de fin de diástole del ventrículo izquierdo.

\section{Evaluar el compromiso del ventrículo derecho}

La evidencia disponible durante los últimos años avala que la función del ventrículo derecho es un determinante del estado funcional, la capacidad de ejercicio y la supervivencia en pacientes con hipertensión arterial pulmonar severa $^{10,19,20}$. Sin embargo, la forma de medir la función del ventrículo derecho y qué parámetros podrían ser clínicamente relevantes, sigue siendo objeto de debate. Por tanto, las mediciones estándar de oro de la función del ventrículo derecho en pacientes con hipertensión arterial pulmonar incluyen necesariamente las estimaciones de la 
contractilidad y la poscarga ${ }^{21}$. En el cateterismo derecho la medición de presiones vasculares pulmonares y el gasto cardíaco, más el cálculo de resistencia vascular pulmonar (RVP) permiten la cuantificación de la severidad de la enfermedad vascular pulmonar, con la limitante de que la RVP proporciona sólo una descripción parcial de todas las fuerzas que se oponen a la salida del flujo desde el ventrículo derecho (o poscarga), y el gasto cardíaco está determinado por las condiciones de precarga, además de las variables intrínsecas en función ventricular y la competencia de las válvulas cardiacas ${ }^{10}$. En la práctica clínica, la determinación del gasto cardiaco y el índice cardíaco se hace típicamente ya sea por el método de termodilución o método Fick (utilizando el principio de Fick).

El método de termodilución consiste en la medición del gasto cardiaco estimado a través de una técnica que permite estimar los cambios de temperatura durante el tiempo y posterior a la administración de una sustancia a temperatura conocida e inferior a la de la sangre ${ }^{22}$. Antes de cada medición es fundamental confirmar la colocación adecuada del catéter de Swan-Ganz, documentando la presencia de una onda de presión de la aurícula derecha desde el puerto proximal del catéter y la visualización fluoroscópica de la punta distal del mismo en la arteria pulmonar. El gasto cardiaco se mide mediante la inyección de $10 \mathrm{ml}$ de solución salina estéril, isotónica $(0,9 \%)$, la cual se inyecta a través del lumen proximal del catéter. En el curso del tiempo se evalúan los cambios de la temperatura reportados por el termistor distal: al conocer la distancia que hay entre el puerto de inyección proximal y el termistor se logra tener los componentes completos de la forma de termodilución. Se aplican tres inyecciones de solución salina en bolo consecutivas para las estimaciones individuales del gasto cardiaco en cada una de ellas, y el valor medio de las tres se utiliza como el valor final para el gasto cardiaco.

\section{Método de Fick indirecto}

El consumo total de oxígeno del cuerpo ( $\left.V^{\prime} \mathrm{O}_{2} / \mathrm{BSA}\right)$, en $\mathrm{ml}$ min $-1 \mathrm{~m}-2$, se estima mediante la fórmula de $F$ ARGE y $M$ IETTINEN $^{23}$ :

Mujeres : $\mathrm{V}^{\prime} \mathrm{O}_{2} / \mathrm{ASC}=\left(138,1-17,04 \times \log _{e}(\mathrm{edad})+0,378 \times \mathrm{FC}\right) / \mathrm{ASC}$

Hombres : $\mathrm{VO}_{2} / \mathrm{ASC}=\left(138,1-11,49 \times \log _{e}(\mathrm{edad})+0,378 \times \mathrm{FC}\right) / \mathrm{ASC}$

Donde $V^{\prime} \mathrm{O}_{2}$ es el consumo de oxígeno en $\mathrm{ml} \min -1$, la edad en años, la frecuencia cardíaca (FC) está en unidades de latidos por minuto, y ASC es la superficie corporal en $\mathrm{m}^{2}$.

Para tal fin se requieren muestras de sangre simultáneas que deben ser tomadas de la aorta y la arteria pulmonar para la determinación de las concentraciones de saturación de oxígeno y hemoglobina. El gasto se calcula según la ecuación de Fick:

$\mathrm{CO}=\mathrm{V}^{\prime} \mathrm{O}_{2} /\left(\mathrm{CaO}_{2}-\mathrm{CvO}_{2}\right)$

Donde se define como $\mathrm{CO} C \mathrm{CO}$ en $\mathrm{L} \cdot \min -1, V^{\prime} \mathrm{O}_{2}$ es el consumo de oxígeno en $\mathrm{L} \min -1 \cdot \mathrm{m}-2, \mathrm{CaO}_{2}$ es el contenido arterial de oxígeno en mg. $\mathrm{L}-1\left(1,36 \times \times \mathrm{Hbg} \mathrm{SaO}_{2}\right)+\left(\mathrm{PaO}_{2}\right.$ $\times 0,003)$ y $\mathrm{C} \mathrm{VO}_{2}$ se define como el contenido de oxígeno venoso mixto en $\mathrm{mg} \cdot \mathrm{L}-1\left(1,36 \times \times \mathrm{Hbg} \mathrm{S} \mathrm{VO}_{2}\right)+\left(P \mathrm{vO}_{2} \times\right.$ $0,003)$. $\mathrm{Hbg}$ es el nivel de hemoglobina en $\mathrm{g} \cdot \mathrm{L}-1, \mathrm{SaO}_{2}$ es la saturación arterial de oxígeno, $\mathrm{PaO}_{2}$ es la tensión de oxígeno arterial en $\mathrm{mm} \mathrm{Hg}, \mathrm{SvO}_{2}$ es la saturación venosa mixta de oxígeno y $\mathrm{PvO}_{2}$ es el oxígeno venoso mixto tensión en $\mathrm{mm}$ $\mathrm{Hg}$.

Existen varios estudios que comparan la superioridad de uno respecto a otros ${ }^{24,25}$. Al parecer existe equilibrio en términos de comparación de uno respecto a otros y en general tiene una buena correlación. Mientras que el patrón de oro de la determinación de $\mathrm{CO}$ es el método directo de Fick, este se utiliza muy poco en la práctica clínica debido a las exigencias técnicas y logísticas que implica la medición directa $V^{\prime} \mathrm{O}_{2}$, que lo hacen poco práctico $^{22}$. Los métodos de Fick y TD, son invasivos y poseen limitaciones inherentes ${ }^{24,25}$. Por ejemplo, TD adolece de precisión debido a las influencias potenciales de la migración del catéter, diferencias en la técnica de inyección (con la variabilidad intra- e interinvestigador), la influencia de las diferentes fases del ciclo respiratorio, entre otros factores ${ }^{25}$. La falta de precisión con termodilución también afecta a la utilidad de su uso como una técnica de referencia cuando se compara con otros métodos de medición de gasto cardiaco $^{25}$. Algunos investigadores han encontrado que la termodilución es menos fiable en el contexto de TR severa y/o baja de gasto cardiaco debido a la pérdida del indicador ${ }^{26,27}$.

El método indirecto Fick también tiene limitaciones que pueden afectar la precisión de medición, en particular por el consumo de oxígeno $\left(V^{\prime} \mathrm{O}_{2}\right)$, dado que es un valor estimado y no obtenido por medición directa. Este valor estimado se puede realizar de forma equívoca llevando a errores en el cálculo del gasto cardiaco. Otros factores que pueden llevar a errores en la medición por el método Fick son: medición de la saturación de oxígeno, niveles de hemoglobina y variables influenciadas por el drenaje venoso bronquial y Tebesio de la saturación venosa mixta ${ }^{25,28}$. Sin embargo, el método indirecto Fick es preciso y fiable en la evaluación de los cambios porcentuales en gasto cardiaco en respuesta a una intervención aguda, ya que el único valor que cambia a menudo en respuesta a la intervención es la saturación venosa mixta, y por tanto los otros factores que intervienen en la estimación se cancelan en el cálculo de los cambios porcentuales en CO. No obstante, en algunos estudios comparativos ${ }^{25}$ existen amplios límites entre uno y otro, hecho que hizo que algunos autores concluyeran que la termodilución y el método Fick no deben considerarse intercambiables.

\section{Conocer un pronóstico de manera objetiva}

Las mediciones hemodinámicas invasivas son bien conocidas como marcadores pronósticos en la hipertensión arterial pulmonar. Tanto la presión de aurícula derecha elevada, como la resistencia vascular pulmonar y el bajo gasto cardiaco son todos indicadores de un pronóstico pobre ${ }^{29,30}$.

En el trabajo clásico de D'Alonso en 1991, el autor mostró cómo un paciente que presenta presión arterial pulmonar media de $40 \mathrm{~mm} \mathrm{Hg}$, presión media de la aurícula derecha de $3,5 \mathrm{~mm} \mathrm{Hg}$ e índice cardíaco de $3,3 \mathrm{~L} / \mathrm{min}$ por $\mathrm{m}^{2}$ ten- 
dría un $85 \%$ probabilidades de sobrevivir durante al menos un año desde el momento en que estos datos fueron recogidos, y una oportunidad de sobrevivir del $72 \%$ durante al menos tres años. En contraste, un paciente que se presenta con presión media de la arteria pulmonar de $80 \mathrm{~mm}$ $\mathrm{Hg}$, presión media de la aurícula derecha de $16 \mathrm{~mm} \mathrm{Hg} \mathrm{e}$ índice cardíaco de 1,3 L/min por $\mathrm{m}^{2}$, tendría un estimado de $46 \%$ de posibilidades de sobrevivir de 1 año y uno del $20 \%$ a 3 años $^{29}$. En otro trabajo se demostró que la reducción de la RVP en un 30\% respecto al valor basal, después de tres meses de tratamiento, es un claro indicador de una mejor supervivencia durante el seguimiento ${ }^{30}$. En el estudio de Tiede et al. un análisis retrospectivo de la hemodinámica de 122 pacientes con hipertensión arterial pulmonar tratados con varios medicamentos específicos, se identificó en el seguimiento hemodinámico, que el tiempo libre de trasplante fue mayor, con una diferencia absoluta del 23,3\% en quienes la RVP disminuyó 2,2 unidades Wood $(p=0,44)$, y adicionalmente existía un incremento en el gasto cardiaco $>0,22 \mathrm{~L} / \min (\mathrm{p}=0,015)^{31}$.

\section{Definir presencia o no de vasorreactividad pulmonar}

El test de vasorreactividad debería hacerse en el momento del cateterismo diagnóstico para identificar pacientes que podrían beneficiarse de una terapia a largo plazo con bloqueadores de los canales de calcio. La recomendación del último Simposio Mundial de Hipertensión Pulmonar es que este test de vasorreactividad pulmonar para la identificación de "respondedores" a los bloqueadores del canal de calcio debería hacerse únicamente en pacientes con hipertensión pulmonar idiopática ${ }^{1}$. El objetivo primario es identificar individuos capacitados para responder a largo plazo con medicamentos calcioantagonistas, lo cual ocurre en el $50 \%$ de los casos. Una respuesta positiva aguda se define como una reducción de la PAPm $>10 \mathrm{~mm} \mathrm{Hg}$ alcanzando un valor absoluto de PAPm $<40 \mathrm{~mm} \mathrm{Hg}$ con un gasto cardiaco que no se modifica o aumenta. Sólo un $10 \%$ de los pacientes con hipertensión arterial pulmonar idiopática cumplirán estos criterios $^{32,33}$. El hallazgo de un individuo respondedor justifica, después del inicio del tratamiento, vigilancia estricta confirmando que existe respuesta hemodinámica óptima porque el impacto benéfico de los calcioantagonistas en el tiempo puede perderse ${ }^{32}$.

\section{Conflicto de intereses}

Ninguno.

\section{Bibliografía}

1. Galiè N, Humbert M, Vachiery JL, et al. 2015 ESC/ERS Guidelines for diagnosis and treatment of pulmonary hypertension. Eur Heart J. 2016 Jan 1;37:67-119. Epub 2015 Aug 29.

2. Simonneau G, Gatzoulis MA, Adatia I, et al. Updated clinical classification of pulmonary hypertension. J Am Coll Cardiol. 2013;62 25 Suppl:D34-41.

3. McGoon MD, Benza RL, Escribano-Subias P, et al. Pulmonary arterial hypertension: epidemiology and registries. J Am Coll Cardiol. 2013 Dec 24;62 25 Suppl:D51-9.
4. Badesch DB, Raskob GE, Elliott CG, et al. Pulmonary arterial hypertension baseline characteristics from the REVEAL Registry. CHEST. 2010;137:376-87.

5. Taichman D, Mandel J. Epidemiology of pulmonary arterial hypertension. Clin Chest Med. 2013;34: 619-37.

6. Champion HC, Michelakis ED, Hassoun PM. Comprehensive invasive and noninvasive approach to the right ventricle-pulmonary circulation unit state of the art and clinical and research implications. Circulation. 2009;120:992-1007.

7. Hoeper MM, Lee SH, Voswinckel R, et al. Complications of right heart catheterization procedures in patients with pulmonary hypertension in experienced centers. J Am Coll Cardiol. 2006;48:2546-52.

8. BarstRJ, McGoon M, Torbicki A, et al. Diagnosis and differential assessment of pulmonary arterial hypertension. J Am Coll Cardiol. 2004;43, 40S-7.

9. Grignola JC. Hemodynamic assessment of pulmonary hypertension. World J Cardiol. 2011;3:10-7.

10. Naeije R, Huez S. Right ventricular function in pulmonary hypertension: physiological concepts. Eur Heart J. 2007; 9 Suppl:H5-9.

11. Chemla D, Castelain V, Hervé P, et al. Haemodynamic evaluation of pulmonary hypertension. Eur Respir J. 2002;20: 1314-31.

12. Kovacs G, Berhold A, Scheidl S, et al. Pulmonary arterial pressure during rest and exercise in healthy subjects: A sistematic review. Eur Resp J. 2009;139:1285-93.

13. Valerio CJ, Schreiber BE, Handler CE, et al. Borderline mean pulmonary artery pressure in patient with systemic sclerosis. Arthritis and Reumatism. 2013;65:1074-84.

14. Soto FJ, Kleczka JF. Cardiopulmonary hemodynamics in pulmonary hypertension: pressure tracings, waveforms, and more. Adv Pulm Hypertension. 2008;7:386-93.

15. Nishimura RA, Carabello BA. Hemodynamics in the cardiac catheterization laboratory of the $21^{\text {st }}$. century. Circulation. 2012;125:2138-50.

16. Halpern SD, Taichman DB. Missclasification of pulmonary hypertension due to reliance on pulmonary capillary wedge pressure rather than left ventricular end- diastolic pressure. Chest. 2009;136:37-43.

17. Ryan JJ, Rich JD, Thiruvoipati T, et al. Current practice for determining pulmonary capillary wedge pressure predisposes to serious errors in the classification of patients with pulmonary hypertension. Am Heart J. 2012;163:589-94.

18. Frantz RP, McGoon MD. Diagnostic dilemmas in pulmonary hypertension. Heart Failure Clin. 2012;8:331-52.

19. Champion HC, Michelakis ED, Hassoun PM. Comprehensive invasive and noninvasive approach to the right ventricle-pulmonary circulation unit: state of the art and clinical and research implications. Circulation. 2009;120:992-1007.

20. Vonk-Noordegraaf A, Haddad F, Chin KM, et al. Right heart adaptation to pulmonary arterial hypertension: physiology and pathobiology. J Am Coll Cardiol. 2013;62:D22-33.

21. Vanderpool RR, Pinsky MR, Naeije R, et al. RV-pulmonary arterial coupling predicts outcome in patients referred for pulmonary hypertension. Heart. 2015;101:37-43.

22. Pavek K, Boska D, Selecky FV. Measurement of cardiac output by thermodilution with constant rate injection of indicator. Circ Res. 1964;15:311-9.

23. LaFarge CG, Miettinen OS. The estimation of oxygen consumption. Cardiovasc Res. 1970;4:23-30.

24. Rich JD, Archer SL, Rich S. Noninvasive cardiac output measurements in patients with pulmonary hypertension. Eur Resp J. 2013;42:125-33.

25. Reuter DA, Huang C, Edrich T, et al. Cardiac output monitoring using indicator-dilution techniques: basics, limits, and perspectives. Anesth Analg. 2010;110:799-811. 
26. Cigarroa RG, Lange RA, Williams RH, et al. Underestimation of cardiac output by thermodilution in patients with tricuspid regurgitation. Am J Med. 1989;86:417-20.

27. Hillis LD, Firth BG, Winniford MD. Analysis of factors affecting the variability of Fick versus indicator dilution measurements of cardiac output. Am J Cardiol. 1985;56:764-8.

28. Fakler U, Pauli C, Hennig M, et al. Assumed oxygen consumption frequently results in large errors in the determination of cardiac output. J Thorac Cardiovasc Surg. 2005;130:272-6.

29. D’Alonzo GE, Barst RJ, Ayres SM, et al. Survival in patients with primary pulmonary hypertension. Results from a national prospective registry. Ann Intern Med. 1991;115:343-9.

30. Sitbon $\mathrm{O}$, Humbert $M$, Nunes $\mathrm{H}$, et al. Long-term intravenous epoprostenol infusion in primary pulmonary hypertension: prognostic factors and survival. J Am Coll Cardiol. 2002;40: 780-8.

31. Tiede H, Sommer N, Milger K, et al. Short term improvement in pulmonary hemodynamics is strongly predictive of long-term survival in patients with pulmonary arterial hypertension. Pull Circ. 2013;3:523-32.

32. Sitbon $O$, Humbert $M$, Jans $X$. Long-term response to calcium channel blockers in idiopathic pulmonary arterial hypertension. Circulation. 2005;111:3105-11.

33. Rich S, Kaufmann E, Levy PS. The effect of high doses of calcium- channel blockers on survival in primary pulmonary hypertension. N Engl J Med. 1992;327:76-81. 